\title{
Calidad de Vida Relacionada con Salud Oral en Mayores de 14 Años en la Comunidad San Juan Bautista, Isla Robinson Crusoe, Chile
}

\author{
Quality of Life Related to Oral Health in Subjects 14 Years or Older, \\ San Juan Bautista Community, Robinson Crusoe Island, Chile
}

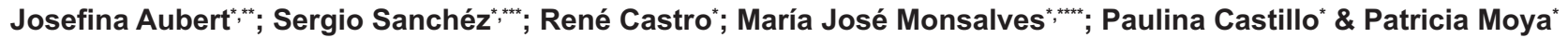

\begin{abstract}
AUBERT, J.; SANCHÉZ, S.; CASTRO, R.; MONSALVES, M. J.; CASTILLO, P. \& MOYA, P. Calidad de vida relacionada con salud oral en mayores de 14 años en la comunidad san juan bautista, isla robinson crusoe, Chile. Int. J. Odontostomat., 8(1):141-145, 2014.

RESUMEN: El aislamiento geográfico y condiciones medioambientales hacen a la población de Juan Fernández altamente vulnerable, siendo relevante conocer la calidad de vida relacionada con salud oral. Se realizó un estudio transversal en 137 habitantes mayores de 14 años. Se aplicó OHIP-14 para medir calidad de vida relacionada con salud oral previo consentimiento informado. El análisis estadístico fue realizado STATA 12.0. Un 51,8\% es población femenina, con una edad media de 42,26 (DE 16,5). Un $91,97 \%$, percibe que la salud oral afecta su calidad de vida, siendo levemente mayor en mujeres. Las molestias psicológicas son una manifestación en $80,29 \%$ de los encuestados. Un $75,91 \%$ percibe incomodidad por dolor en su boca, $62,77 \%$ manifiesta problemas de incapacidad psicológica. Existe mayor impacto en la calidad de vida relacionada con salud oral a medida que aumenta la edad. Por cada año de vida, el valor del OHIP-14 aumenta 0,07 (IC:95\% 0,04-0,1). Los resultados obtenidos sugieren la necesidad de generar políticas públicas que consideren la salud oral desde una perspectiva integral, con énfasis en poblaciones altamente vulnerables considerando la mejora en la calidad de vida como un resultado a esperar.
\end{abstract}

PALABRAS CLAVE: salud oral, calidad de vida, Oral Health Impact Profile.

\section{INTRODUCCIÓN}

El archipiélago de Juan Fernández es parte del territorio Chileno ubicado en el Pacífico Sur (División de Planificación regional Valparaíso, 2012). La población total es de 792 personas con una mediana de edad de 34 años. El 78,66\% de la población es mayor de 15 años y un 5,93\% pertenece a alguna etnia (INE, 2014). El aislamiento geográfico y las condiciones medioambientales en que vive esta comunidad los determina como un grupo humano vulnerable a diversas enfermedades, incluyendo las bucales.

En la actualidad, las enfermedades orales constituyen un problema importante de salud a nivel mundial y nacional, que contribuyen con una alta carga de enfermedad y por tanto con un peso importante en relación a la calidad de vida general de los individuos (Marcenes et al., 2013). En Chile, éstas siguen siendo altamente prevalentes produciendo una alta carga de enfermedad en todos los grupos etarios, con gradientes diferenciadas principalmente por edad, nivel socioeconómico y lugar de procedencia (MINSAL, 2007). Para los adultos toma gran relevancia principalmente porque las condiciones orales se caracterizan por ser patologías crónicas, de larga data y lenta progresión. A esto se suma la falta de políticas públicas que hayan abordado a este grupo etario durante su ciclo vital (MINSAL, 2007; Monsalves, 2012).

Los estudios de calidad de vida han sido incorporados en la literatura científica en forma creciente, con el fin de abordar el concepto de Salud en todas sus dimensiones y relacionarlo a los indicadores epidemiológicos (MINSAL, 2006).

* Académico Facultad de Odontología, Universidad Finis Terrae, Santiago, Chile.

** Alumna del Magíster en Salud Pública, Universidad de Chile, Santiago, Chile.

*** Decano Facultad de Odontología, Universidad Finis Terrae, Santiago, Chile.

**** Doctorando en Salud Pública, Universidad de Chile, Santiago, Chile. 
La evidencia refiere que la salud oral es un componente significativo en la calidad de vida general de los individuos, con importantes implicancias en el estado de salud, sin embargo a nivel país no hay estudios de calidad de vida relacionada a salud oral en base a muestras representativas (Kandelman et al., 2008). La II Encuesta de calidad de vida y salud incluyó una pregunta en relación a salud oral, donde el $43,6 \%$ de los encuestados indicó que rara vez o nunca su salud bucal, es decir, el estado de sus dientes y encías, afecta su calidad de vida (MINSAL, 2006).

Existen herramientas validadas que permitan evaluar tanto la calidad de vida relacionada con salud general, como en relación a salud oral, las cuales permiten medir el impacto real de las intervenciones en salud en la calidad de vida autoreportada por las personas (Misrachi \& Espinoza, 2005).

Una de las herramientas más utilizadas en salud bucal es el Oral Health Impact Profile (OHIP), el cual mide cómo influyen las enfermedades orales en el impacto social de sentirse bien (Slade, 1997). Es el instrumento más utilizado para evaluar calidad de vida relacionada con salud oral en población joven y adulta en el mundo. Esta encuesta fue validada en Chile en población adolescente (López \& Baelum, 2009).

Dado el contexto socio-geográfico y vulnerabilidad de ésta población, es de relevancia conocer la percepción de la calidad de vida relacionada con salud oral, con el fin de establecer una línea de base para futuras investigaciones locales y nacionales.

\section{MATERIAL Y MÉTODO}

Se realizó un estudio de corte transversal en una muestra de 137 personas mayores de 14 años que vive en el poblado de San Juan Bautista, Isla Robinson Crusoe del Archipiélago de Juan Fernández. Las variables incluidas en el estudio fueron sexo, edad y percepción de calidad de vida relacionada con salud oral. Se aplicó el instrumento OHIP-14 (versión simplificada del OHIP-49) para medir calidad de vida relacionada con salud oral. Éste fue auto-administrado, previo consentimiento informado. Se excluyeron a personas analfabetas y con limitaciones cognitivas.

El cuestionario OHIP-14 contempla siete dimensiones para medir la calidad de vida relacionada con salud oral, que incluye 2 preguntas por cada dimen- sión. La variable de resultado es continua siendo el rango esperable de 0 a 28 puntos. Para su análisis el valor final de cada encuestado se categoriza como "bajo impacto" $=0$ (buena percepción de la calidad de vida relacionada con salud oral) y de "alto impacto" > a 0 (mala percepción de la calidad de vida relacionada con salud oral) (Slade; López \& Baelum).

Las encuestas se tabularon en una planilla Excel y luego se realizó el análisis estadístico mediante Stata 12.0. Este análisis se dividió en dos etapas, la primera de análisis descriptivo de los datos valorizando cada dimensión del índice OHIP-14 según impacto, se calculó la media para cada dimensión con los ponderadores establecidos por el instrumento (Slade; López \& Baelum). Además, se calculó el IC al 95\% para las medias de cada dimensión diferenciadas por sexo, las cuales se contrastaron mediante T-test. En una segunda etapa se realizó el cruce de las variables edad y calidad de vida mediante regresión lineal. Finalmente, se dicotomizaron los puntajes (alto y bajo impacto), y se analizó por sexo mediante $\mathrm{Chi}^{2}$. Los principales resultados se resumen en tablas de frecuencia y se graficó la regresión lineal.

\section{RESULTADOS}

Un $51,82 \%$ de la muestra es de sexo femenino. La media de edad fue de 42,26 años (DS 16,5), siendo similar entre hombres y mujeres $(p>0.05)$.

La media ponderada del OHIP-14 fue de 3,67 (IC 95\%: 3,1-4,24) siendo mayor en las mujeres $(p=0,05)$. En las dimensiones dolor físico, molestias psicológicas e incapacidad psicológica se observaron diferencias estadísticamente significativas en la media de calidad de vida y sexo (Tabla I).

La proporción de individuos que percibe que la salud oral afecta su calidad de vida fue de un $91,97 \%$, siendo levemente mayor en las mujeres $(p=0,85)($ Tabla II).

En la dimensión limitación funcional, el 48,99\% de los encuestados manifiesta tener problemas en la fonación y sensación del gusto, sin diferencias estadísticamente significativas por sexo. Un $75,91 \%$ percibe incomodidad por dolor en su boca o al comer algunos alimentos debido a problemas con sus dientes, boca o prótesis, siendo mayor en mujeres $(p=0,01)$. 
AUBERT, J.; SANCHÉZ, S.; CASTRO, R.; MONSALVES, M. J.; CASTILLO, P. \& MOYA, P. Calidad de vida relacionada con salud oral en mayores de 14 años en la comunidad san juan bautista, isla robinson crusoe, Chile. Int. J. Odontostomat., 8(1):141-145, 2014.

Tabla I. Relación entre la calidad de vida relacionada con salud oral y sexo.

\begin{tabular}{lrrrr}
\hline \multirow{2}{*}{ Dimensión } & Puntaje & \multicolumn{2}{c}{ Puntaje por sexo } & \multirow{2}{*}{ Valor p } \\
\cline { 2 - 4 } & Promedio (IC 95\%) & Femenino (IC 95\%) & Masculino (IC 95\%) & \\
\hline Limitación Funcional & $0,32(0,25-0,39)$ & $0,33(0,23-0,43)$ & $0,31(0,21-0,42)$ & 0,42 \\
Dolor físico & $0,64(0,55-0,74)$ & $0,74(0,62-0,87)$ & $0,53(0,39-0,67)$ & $\mathbf{0 , 0 1 *}$ \\
Molestias sicológicas & $0,78(0,67-0,88)$ & $0,87(0,72-1,02)$ & $0,68(0,54-0,83)$ & $\mathbf{0 , 0 3 *}$ \\
Incapacidad física & $0,38(0,29-0,47)$ & $0,41(0,27-0,55)$ & $0,35(0,23-0,46)$ & 0,25 \\
Incapacidad sicológica & $0,53(0,43-0,62)$ & $0,62(0,47-0,77)$ & $0,43(0,31-0,54)$ & $\mathbf{0 , 0 2 *}$ \\
Incapacidad social & $0,27(0,19-0,35)$ & $0,32(0,20-0,44)$ & $0,21(0,10-0,33)$ & 0,09 \\
Obstáculos & $0,34(0,26-0,43)$ & $0,38(0,26-0,50)$ & $0,31(0,19-0,42)$ & 0,2 \\
OHIP- 14 & $3,67(3,1-4,24)$ & $4,11(3,27-4,94)$ & $3,2(2,43-3,97)$ & $\mathbf{0 , 0 5 *}$ \\
\hline
\end{tabular}

*= p estadísticamente significativo; ${ }^{*}=I C 95 \%$ consistente, rechaza hipótesis.

Tabla II. Relación entre percepción de mala calidad de vida relacionada con salud oral por dimensiones, según sexo.

\begin{tabular}{lccc}
\hline \multirow{2}{*}{ Dimensión } & \multicolumn{2}{c}{ Sexo (\%) } & \multirow{2}{*}{ Valor p } \\
\cline { 2 - 3 } & Femenino & Masculino & \\
\hline Limitación Funcional & 50,75 & 49,25 & 0,80 \\
Dolor físico & 57,69 & 42,31 & $\mathbf{0 , 0 1 *}$ \\
Molestias sicológicas & 54,55 & 45,45 & 0,19 \\
Incapacidad física & 50,0 & 50,0 & 0,68 \\
Incapacidad sicológica & 53,49 & 46,51 & 0,61 \\
Incapacidad social & 60,42 & 39,58 & 0,13 \\
Obstáculos & 52,38 & 47,62 & 0,90 \\
OHIP-14 & 51,59 & 48,41 & 0,85 \\
\hline
\end{tabular}

* $=$ p estadísticamente significativo; rechaza hipótesis nula.

Las molestias psicológicas son una manifestación en $80,29 \%$ de la muestra, siendo similar en ambos grupos. Un 48,18\% percibe limitación en la masticación como incapacidad física, no existiendo diferencias por sexo. Un $62,77 \%$ manifiesta problemas de incapacidad psicológica como la autoestima y relación con los otros, el 35,04\% percibe problemas de interacción social e irritabilidad. Un 45,99\% siente incapacidad para realizar las actividades

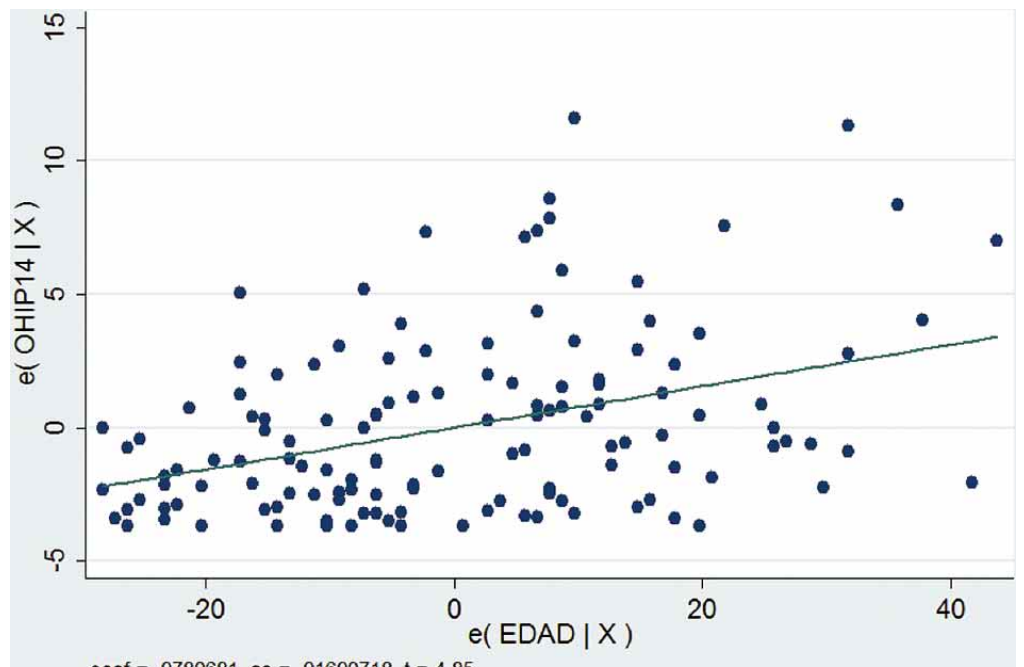

Fig. 1. Regresión lineal del OHIP-14 en relación a la edad. de la vida diaria a causa de sus dientes, boca o prótesis.

En base al análisis mediante regresión lineal se observó que por cada año de vida el valor OHIP-14 aumenta 0,07 puntos (IC:95\%, 0,04-0,1) (Fig. 1).

\section{DISCUSIÓN}

El concepto de calidad de vida relacionado a salud oral que se ha acuñado en los últimos años requiere considerar para su evaluación, no sólo factores tales como el malestar, dolor o alteraciones de la función, sino también debe incluir aspectos emocionales y funciones sociales asociadas a la salud oral (Locker \& Quiñonez, 2011; Sheiman, 2005).

Se ha referido que la salud oral es un elemento importante de la salud general y de la calidad de vida de los individuos, situación que se ve reflejada en el presente estudio, ya que las personas perciben que existe un asociación entre su calidad de vida y su salud oral (Slade).

Tanto en la Encuesta de Calidad de Vida (ENCAVI), como en la Encuesta Nacional de Salud (ENS) se establece una asociación entre la calidad de vida y la edad (MINSAL, 2006; MINSAL, 2010). Mientras mayor es la edad, peor es la percepción de calidad de vida, siendo coherente con los resultados obtenidos en relación a calidad de vida relacionada a salud bucal en este estudio. 
La dimensión que parece influir en mayor medida, de forma negativa en la percepción de la calidad de vida relacionada con la salud oral, es el "dolor o incomodidad" y está asociada a la variable sexo. En este estudio se observaron diferencias significativas en algunas dimensiones, siendo más afectada en todas ellas el sexo femenino, lo que es concordante con la evidencia actual (MINSAL, 2006; MINSAL, 2010).

Mediante este estudio es posible estimar el impacto negativo que tienen las condiciones de salud bucal sobre la calidad de vida de las personas, lo cual hace de este tema un problema relevante para la Salud Pública e incita a cuestionarse cuál es el peso de la autopercepción de la salud bucal en la calidad de vida relacionada con salud y en la calidad de vida general. Ya que en la Encuesta II de calidad de vida y salud revela mediante los resultados obtenidos, que la salud bucal no tendría un peso significativo en la calidad de vida general de los individuos. Sin embargo, se sugiere evaluar si mediante una pregunta es posible inferir la influencia de la salud bucal en la calidad de vida general.
Los resultados obtenidos se presentan como un desafío para la Salud Pública de nuestro país, lo que sugiere generar políticas públicas que consideren la salud oral desde una perspectiva integral, con énfasis en poblaciones altamente vulnerables. La OMS recomienda que los países desarrollen políticas públicas basadas en la integración de la prevención y promoción de salud. En estos análisis no sólo deben considerarse aspectos biológicos sino también, los contextos de los individuos, valores culturales, creencias asociadas e incluso los entornos familiares (Kandelman et al.). Aspectos fundamentales a considerar en la inclusión de nuevas políticas, estrategias y programas en relación a salud oral en Chile.

\section{AGRADECIMIENTOS}

Los autores agradecen a la Universidad Finis Terrae y a la Armada de Chile por el apoyo y tiempo destinado para la elaboración y ejecución de esta investigación.

AUBERT, J.; SANCHÉZ, S.; CASTRO, R.; MONSALVES, M. J.; CASTILLO, P. \& MOYA, P. Quality of Life Related to Oral Health in Subjects 14 Years or Older, San Juan Bautista Community, Robinson Crusoe Island, Chile. Int. J. Odontostomat., 8(1):141-145, 2014.

ABSTRACT: Geographic isolation and environmental conditions make the population of Robinson Crusoe Island, in the Juan Fernández archipielago highly vulnerable. It is therefore relevant to know the quality of life related to oral health (QOLRTO). In this report a cross sectional study in 137 inhabitants over 14 years of age was carried out. OHIP-14 was applied to measure QOLRTO, after informed consent was obtained. Statistical analysis was made in STATA 12.0. The population is $51.8 \%$ female, mean age 42.26 (SD 16.5) being similar in both sexes. Of the subjects studied, $91.97 \%$ perceived that oral health affects their quality of life, being slightly higher in women. Psychological distress was a manifestation in $80.29 \%$ of subjects, while $75.91 \%$ perceived discomfort and mouth pain, and $62.77 \%$ reported psychological disability issues. There is a greater impact of oral health issues with increasing age. For each year of life, the value of the OHIP-14 increased $0.07(\mathrm{Cl} 95 \%, 0.04$ to 0.1$)$. The results suggest that public health policies that include a comprehensive oral health perspective, with emphasis on highly vulnerable populations should be considered.

KEY WORDS: oral health, quality of life, Oral Health Impact Profile.

\section{REFERENCIAS BIBLIOGRÁFICAS}

División de Planificación y Desarrollo, Gobierno Regional Región de Valparaíso. Estrategia regional de desarrollo Región Valparaíso 2020. División de Planificación y Desarrollo, Gobierno Regional Región de Valparaíso, 2012. Disponible en: http://fic.gorev.cl/descargas/2013/Estrategia-Regional-de-Desarrollo2020.pdf

Instituto Nacional de Estadísticas (INE). Chile proyecciones y estimaciones de población. 2014. Disponible en: http:/ /www.ine.cl/canales/chile estadistico/ demografia_y_vitales/demografia/demografia.php

Kandelman, D.; Petersen, P. E. \& Ueda, H. Oral health, general health, and quality of life in older people. Spec. Care Dentist., 28(6):224-36, 2008.

Locker, D. \& Quiñonez, C. To what extent do oral disorders compromise the quality of life? Community Dent. Oral Epidemiol., 39(1):3-11, 2011. 
AUBERT, J.; SANCHÉZ, S.; CASTRO, R.; MONSALVES, M. J.; CASTILLO, P. \& MOYA, P. Calidad de vida relacionada con salud oral en mayores de 14 años en la comunidad san juan bautista, isla robinson crusoe, Chile. Int. J. Odontostomat., 8(1):141-145, 2014.

López, R. \& Baelum, V. Spanish version of the Oral Health Impact Profile (OHIP-Sp). BMC Oral Health, 6:11, 2006.

Marcenes, W.; Kassebaum, N. J.; Bernabé, E.; Flaxman, A.; Naghavi, M.; Lopez, A. \& Murray, C. J. Global Burden of Oral Conditions in 1990-2010: A Systematic Analysis. J. Dent. Res., 92(7):592-7, 2013.

Ministerio de Salud (MINSAL). I/ Encuesta de calidad de vida y salud Chile 2006. Santiago, Ministerio de Salud, Gobierno de Chile, 2006. Disponible en: http://epi.minsal.cl/ wp-content/uploads/2012/07/Informe-Final-Encuesta-deCalidad-de-Vida-y-Salud-2006.pdf

Ministerio de Salud (MINSAL). Informe Final Estudio de carga de enfermedad y carga atribuible, Chile 2007. Santiago, Ministerio de Salud, Gobierno de Chile, 2007. Disponible en: http://epi.minsal.cl/epi/html/invest/ cargaenf2008/Informe\%20final\%20carga_Enf_2007.pdf

Ministerio de Salud (MINSAL). Segunda Encuesta Nacional de Salud. Santiago, Ministerio de Salud, Gobierno de Chile, 2010. Disponible en: http://web.minsal.cl/portal/url/ item/bcb03d7bc28b64dfe040010165012d23.pdf

Misrachi, C. \& Espinoza, I. Utilidad de las mediciones de vida relacionada con la Salud. Rev. Dent. Chile, 96(2):2835, 2005

Monsalves, M. J. La odontología que vivimos y no queremos. Rev. Chil. Salud Pública, 16(2):241-6, 2012.

Sheiham, A. Oral health, general health and quality of life. Bulletin of the World Health Organization. Bull. World Health Organ., 83(9):644-5, 2005.

Slade, G. D. Measuring Oral Health and Quality of Life. Chapel Hill, University of North Carolina, Dental Ecology, 1997.
Dirección para Correspondencia:

Josefina Aubert

Facultad de Odontología

Universidad FinisTerrae

Avda. Pedro de Valdivia 1509

Providencia, Santiago

CHILE

Email: jaubert@uft.cl

Recibido : 22-10-2013

Aceptado: 23-12-2013 